\title{
Triiodothyronine modulates the expression of aquaporin-8 in rat liver mitochondria
}

\author{
Giuseppe Calamita, Maria Moreno ${ }^{1}$, Domenico Ferri², Elena Silvestri ${ }^{1}$, Patrizia Roberti, Luigi Schiavo \\ Patrizia Gena, Maria Svelto and Fernando Goglia'
}

Department of General and Environmental Physiology, Università degli Studi di Bari, Via Amendola 165/A, 70126 Bari, Italy

${ }^{1}$ Department of Biological and Environmental Sciences, University of Sannio, Via Port'Arsa 11, 82100 Benevento, Italy

${ }^{2}$ Department of Zoology, University of Bari, Bari, Italy

(Requests for offprints should be addressed to G Calamita; Email: calamita@biologia.uniba.it)

\begin{abstract}
The recent identification of aquaporin-8 (AQP8), an aquaporin (AQP) channel permeable to water and ammonia, in the inner membrane (IMM) of rat liver mitochondria suggested a role for such AQP in the hydration state and the metabolic function of mitochondria. Since thyroid hormone triiodothyronine (T3) is known to modulate both the shape and the metabolic activities of liver mitochondria, it was interesting to investigate the expression and distribution of AQP8 as well as the osmotic water permeability of the IMM in liver mitochondria from rats in different thyroid states. By semi-quantitative reverse transcriptase (RT)-PCR, when compared with the euthyroid counterpart, the levels of hepatic AQP8 mRNA significantly increased in the
\end{abstract}

hypothyroid state, whereas they were strongly decreased after administration of T3. A similar pattern was seen at the protein level by immunoblotting mitochondrial membranes. The upregulation of mitochondrial AQP8 in the hypothyroid liver was confirmed by immunogold electron microscopy. Stopped-flow light scattering with IMM vesicles showed no significant differences in terms of osmotic water permeability among the IMMs in the various thyroid states. Overall, our data indicate that the T3 modulation of the AQP8 gene is a rapid downregulation of transcription. Modulation of hepatic AQP8 expression may be relevant to the regulation of mitochondrial metabolism by thyroid hormones.

Journal of Endocrinology (2007) 192, 111-120

\section{Introduction}

Triiodothyronine (T3) exerts significant actions on energy metabolism, with mitochondria being a major target for its effects (Soboll 1993). Extensive changes occur in the mitochondrial compartment in response either to thyroid hormone administration or to physiological states modulating thyroid gland activity (e.g. cold exposure, aging, dietary changes; Goglia et al. 1999). Indeed, alterations to the thyroid state of animals have considerable effects on the synthesis (Roodyn 1965, Goglia et al. 1988), the turnover (Gross 1971), and the functional capacity of mitochondrial components. Liver mitochondria from hypothyroid rats have a decreased activity of membrane-associated electron transport enzymes and anion carriers (Paradies et al. 1994), a failure that has been ascribed to a lower expression of their corresponding proteins as well as to changes in the composition of the inner membrane (Soboll et al. 1994, Schonfeld et al. 1997). Thyroid hormones are also known to modulate both shape and metabolic efficiency of mitochondria (Jakovcic et al. 1978, Goglia et al. 1988). However, although marked differences in the shape and the number of the cristae have been reported in the liver mitochondria of rats in different thyroid states
(Jakovcic et al. 1978, Goglia et al. 1989), the molecular mechanisms underlying T3 modulation of mitochondrial morphology remain mostly elusive. A clue to understanding such mechanisms relates to the fact that mitochondria are well-behaved osmometers and that their shape is influenced by the movement of water accompanying the net transport of solutes into and out of their matrix (Beavis et al. 1985).

Interestingly, an aquaporin (AQP) water channel, aquaporin-8 (AQP8), has recently been identified in the inner mitochondrial membrane (IMM) of liver and other tissues (Ferri et al. 2003, Calamita et al. 2005, Lee et al. 2005) by providing valuable insights into the knowledge of the homeostatic mechanisms underlying mitochondrial volume homeostasis and shape plasticity. AQP8 immunostaining was found to vary among liver mitochondria with heavy mitochondria being the more reactive ones, an observation that led to the speculation that AQP8-mediated water transport could be important for rapid expansion of mitochondrial volume (Calamita et al. 2005). Therefore, in spite of their high surface-to-volume ratio, a feature that can justify millisecond osmotic equilibration (Yang et al. 2006a), mitochondria possess facilitated pathways for water diffusion other than the AQP8 water channels (Calamita et al. 2006). 
In rat, liver AQP8 has also been suggested to play a role in amino acid metabolism and detoxification of ammonia by mediating the mitochondrial uptake of $\mathrm{NH}_{4}^{+}$to supply the urea cycle (Holm et al. 2005), a process known to be influenced by the thyroid states (Marti et al. 1988, Hayase et al. 1991). However, the physiological significance of the AQP8facilitated ammonia transport was questioned in a recent work by Yang et al. (2006b).

Aquaporins (AQP9 and AQP1) have already been reported to be directly modulated by $\mathrm{T} 3$ at a transcriptional level in porcine liver (Caperna et al. 2007) and rabbit developing kidney (Mulder et al. 2003) respectively. However, no information is yet available about possible effects exerted by the different thyroid states on the expression of AQP8 in mitochondria.

The aim of the present work was to investigate the effect of thyroid hormone on the expression and distribution of AQP8 water channels in the liver mitochondria of rats in various thyroid states.

\section{Materials and Methods}

\section{Animals}

Male Wistar rats (250-300 g; Charles River, Lecco, Italy) were kept one per cage in a temperature-controlled room at a rat thermoneutral temperature of $28{ }^{\circ} \mathrm{C}$ under a $12 \mathrm{~h}$ light: $12 \mathrm{~h}$ darkness cycle. A commercial mash and water were available ad libitum. Three groups of rats (each consisting of three animals) were used throughout: euthyroid (referred to as $\mathrm{N}$ ), hypothyroid (referred to as Hypo), and T3-treated hypothyroid rats (referred to as Hypo + T3). Hypothyroidism was induced by the i.p. administration of propylthiouracil (1 mg/100 g body weight) for 4 weeks together with a weekly i.p. injection of iopanoic acid $(6 \mathrm{mg} / 100 \mathrm{~g}$ body weight; Lanni et al. 1996, Moreno et al. 1997). This treatment allowed us to obtain hypothyroid rats with low thyroid-hormone levels and an inhibition of all three deiodinase enzymes (Moreno et al. 1997). T3 was chronically administered by giving a daily i.p. injection of $15 \mu \mathrm{g}$ T3/100 g body weight to hypothyroid rats for 7 days, while the control hypothyroid rats and the euthyroid ones received saline. The doses of T3 used in the present study were within the average range of those used in most studies to obtain a mild hyperthyroid state without inducing a syndrome of hypermetabolism (Freake et al. 1989). This animal model of Hypo + T3 allows us to exclude the effects of other active iodothyronines putatively derived from T3 peripheral metabolism after T3 injection, as all deiodinase enzymes were strongly inhibited.

At the end of the T3 or saline treatment ( $24 \mathrm{~h}$ after the last injection), rats were anesthetized and killed by decapitation. Trunk blood was collected and serum isolated to allow analysis of hormone levels using specific RIAs (ICN Pharmaceuticals, Diagnostic Division, New York, NY, USA). Livers were excised, weighed, and immediately frozen in liquid nitrogen and stored at $-80{ }^{\circ} \mathrm{C}$ for later processing. All experiments were performed in accordance with European Union general guidelines regarding animal experiments and were approved by our institutional committee for animal care. For time-course experiments, Hypo rats were acutely injected with $25 \mu \mathrm{g}$ T3/100 g body weight and killed after $6,12,24$, and $48 \mathrm{~h}$.

\section{Preparation of mitochondria}

Mitochondria were isolated as previously described (Lanni et al. 1996, Calamita et al. 2005). Briefly, at the end of the T3 or saline treatments, livers were homogenized with a PotterElvehjem homogenizer (four strokes in $1 \mathrm{~min}$ at 500 r.p.m.) in an isolation medium consisting of $220 \mathrm{mM}$ mannitol, $70 \mathrm{mM}$ sucrose, $20 \mathrm{mM}$ Tris- $\mathrm{HCl}$ (pH 7•4), $1 \mathrm{mM}$ EDTA, and $5 \mathrm{mM}$ EGTA. The homogenate was centrifuged at $500 \mathrm{~g}$ for $10 \mathrm{~min}$ at $4{ }^{\circ} \mathrm{C}$ and the pellet consisting of nuclei and unbroken cells was discarded; the resulting supernatant was centrifuged at $3000 \mathrm{~g}$ for $10 \mathrm{~min}$ at $4{ }^{\circ} \mathrm{C}$ and the related pellet was washed twice before being resuspended in isolation medium. The $3000 \mathrm{~g}$ supernatant was then used to prepare the plasma membrane $(17000 \mathrm{~g})$ and microsomal $(100000 \mathrm{~g})$ fractions. Mitochondria for immunoblot analyses were resuspended in isolation medium to which a cocktail of protease inhibitors had been added $(1 \mathrm{mM}$ phenylmethylsulphonyl fluoride, $1 \mathrm{mM}$ leupeptin, and $1 \mathrm{mM}$ pepstatin $\mathrm{A}$ ).

\section{Mitochondrial respiration rate measurements}

Mitochondrial oxygen consumption was measured polarographically using a Clark-type electrode. The measurements were carried out in duplicate using succinate $(6 \mathrm{mM})$ as substrate. The analyses were performed in a final volume of $0.5 \mathrm{ml}$ of $80 \mathrm{mM} \mathrm{KCl}, 50 \mathrm{mM}$ Hepes ( $\mathrm{pH} 7 \cdot 2-7 \cdot 4), 1 \mathrm{mM}$ EGTA, $5 \mathrm{mM} \mathrm{K}_{2} \mathrm{HPO}_{4}$, and $0 \cdot 3 \%$ BSA (w/v), both in the absence (state 4 respiration) and in the presence (state 3 respiration) of $\operatorname{ADP}(300 \mu \mathrm{M})$ at $37{ }^{\circ} \mathrm{C}$. Values for respiratory control ratios (RCRs; state 3 rate divided by state 4 rate) were calculated according to the method of Estabrook (1967).

The IMM vesicles were prepared from the $3000 \mathrm{~g}$ mitochondria as previously reported (Calamita et al. 2005). The purity of the vesicles was assessed by immunoblotting the enrichment of the IMM protein marker prohibitin as previously described (Calamita et al. 2005).

\section{RT-PCR analysis}

Total RNA was prepared from frozen tissue samples using the Trizol kit according to the manufacturer's protocol (Invitrogen). One microgram of total RNA was reverse-transcribed as previously reported (Moreno et al. 2003). The primers used for the RT-PCR analyses had the following sequences: $\beta$-actin sense, $5^{\prime}$-TTGTAACCAACTGGGACGAT-3'; $\beta$-actin antisense, 5' ${ }^{\prime}$-TAATGTCACGCACGATTTCC-3'; AQP8 sense (mAQP8-30cons) 5'-GGTGGACACTTCAACCCTGC-3'; 
and AQP8 antisense (mAQP8-31 cons) 5'-CCCAGCCAGTAGATCCAATG-3'. Separation of the PCR products was performed on a $2 \%$ agarose gel containing ethidium bromide. Reverse-image signals of the RT-PCR bands were quantified by means of a Bio-Rad Molecular Imager FX using the supplied software (Bio-Rad Laboratories). The optical densities of the AQP8 bands were normalized with respect to the non-regulated $\beta$-actin bands.

\section{Immunoblot analysis}

Aliquots $(60 \mu \mathrm{g}$ protein) of isolated mitochondria prepared as above were heated to $90{ }^{\circ} \mathrm{C}$ and electrophoresed in an SDS $13 \%$ acrylamide gel (Mighty Small II, Amersham Biosciences) using a low molecular weight protein ladder (Amersham Biosciences). The resolved proteins were transferred electrophoretically onto nylon membranes that were blocked in 5\% (w/v) low fat milk in blocking buffer (20 mM Tris- $\mathrm{HCl}$ (pH 7.5), 0.15 M NaCl, 1\% Triton $\mathrm{X}-100)$ for $1 \mathrm{~h}$, and further incubated with affinity purified rabbit antibodies against an $\mathrm{N}$-terminal peptide of rat AQP8 (Ferri et al. 2003) in a final concentration of $1 \mu \mathrm{g} / \mathrm{ml}$ blocking solution. Horseradish peroxidase antirabbit IgG-treated membranes (anti-rabbit IgG peroxidase antibody; Sigma) were developed by luminal chemiluminescence (ECL-Plus, Amersham Biosciences).

\section{Immunogold electron microscopy studies}

Four animals per each condition were used for the study. Samples of mitochondrial pellets obtained as above were fixed in a mixture of 3\% paraformaldehyde and $1 \%$ glutaraldehyde in $0.1 \mathrm{~mol} / 1 \mathrm{PBS}$ at $\mathrm{pH} 7 \cdot 4$ for $4 \mathrm{~h}$ at $4{ }^{\circ} \mathrm{C}$ and then processed for immunogold electron microscopy as reported in our previous work (Calamita et al. 2005). Immunolabeling controls were performed by omitting the AQP8 antibody. At least 15 images were obtained from each pellet of freshly isolated mitochondria, while morphometric analysis was carried out by counting the number of immunogold particles over an overall micrograph surface of about $3000 \mu \mathrm{m}^{2}$.

\section{Stopped-flow light scattering}

The size of the IMM vesicles to be used for the stopped-flow measurements was determined both with a N5 Submicron Particle Size Analyzer (Beckman Coulter Inc., Palo Alto, CA, USA) and by morphometric analysis of electron micrographs. The time course of vesicular volume change was followed from changes in the intensity of scattered light at a wavelength of $450 \mathrm{~nm}$ using a Jasco FP-6200 (Jasco, Tokyo, Japan) stopped-flow reaction analyzer, which has a $1.6 \mathrm{~ms}$ dead time and $99 \%$ mixing efficiency in $<1 \mathrm{~ms}$. The sample temperature $\left(20{ }^{\circ} \mathrm{C}\right)$ was controlled by a circulating water bath. Stopped-flow measurements were performed by following the same experimental conditions and biophysical formulation described in our previous work (Calamita et al. 2005).
The data were fitted to a single exponential function. The osmotic water permeability coefficient $\left(P_{\mathrm{f}}\right)$, an index reflecting the osmotic water permeability of the vesicular membrane, was calculated using the van Heeswijk \& van Os (1986) equation: $P_{\mathrm{f}}=K_{\exp } \cdot V_{0} / A_{\mathrm{v}} \cdot V_{\mathrm{w}} \cdot \Delta C$, where $K_{\exp }$ is the fitted exponential rate constant, $V_{0}$ is the initial mean of vesicle volume, $A_{\mathrm{v}}$ is the mean vesicle surface, $V_{\mathrm{w}}$ is the molar volume of water, and $\Delta C$ is the osmotic gradient. The medium osmolarity was verified by freezing point depression, using a Halbmikro Osmometer (Knauer, Berlin, Germany). In some experiments, the IMM vesicles were incubated for 5 min with $300 \mu \mathrm{M} \mathrm{HgCl}$, a compound known to block most mammalian AQPs, including AQP8 (Ishibashi et al. 1997, Liu et al. 2006). To verify the blocking action of the $\mathrm{Hg}^{2+}$ ion, the $\mathrm{HgCl}_{2}$ treatment of the vesicles was followed by a $15-\mathrm{min}$ exposure to $10 \mathrm{mM}$ reducing agent $\beta$-mercaptoethanol.

\section{Statistical analysis}

All experiments were performed at least in triplicate. Means \pm s.e. were calculated based on three to five independent preparations. Data were analyzed statistically using Student's $t$-test. Results were considered statistically significant when $P<0 \cdot 05$.

\section{Results}

Respiratory parameters of liver mitochondria in the various thyroid states

As a first step, in order to ascertain the effectiveness of the experimental treatment, we assessed the thyroid state of each animal by measuring the serum levels of total triiodothyroxine (TT3) and total thyroxine (TT4). As expected, the T3 and T4 levels were significantly lower in hypothyroid rats than in euthyroid ones (Table 1). T3 administration to hypothyroid rats increased TT3 levels by about 1.5- and 8-fold in comparison with the euthyroid and hypothyroid rats respectively (Table 1$)$.

To further confirm, at cellular level, the thyroid state of the animals, we next measured the respiratory parameters of the liver mitochondria of the N, Hypo-, and Hypo + T3 rats. As expected, using succinate as substrate, both state 3 and state 4 oxygen consumption rates were significantly reduced in liver mitochondria from hypothyroid rats in comparison with the euthyroid values (Table 1). T3 administration to hypothyroid animals restored the values observed in euthyroid rats (Table 1). No change in the RCR was detected between the three groups. These metabolic parameters were also in line with the thyroid state of the animals used throughout. 
Table 1 Liver mitochondria respiration state 3 and state 4 rates, RCR and total serum T3 (TT3) and T4 (TT4) in rats in different thyroid states. Results are presented as mean \pm s.E.M. of values from three rats in each group. Determinations were done in triplicate.

\begin{tabular}{|c|c|c|c|c|c|}
\hline & State 3 & State 4 & RCR & TT3 & TT4 \\
\hline \multicolumn{6}{|c|}{ Animal group } \\
\hline Eu- & $220 \pm 10$ & $37 \pm 3$ & $5 \cdot 9 \pm 0 \cdot 3$ & $0 \cdot 85 \pm 0 \cdot 04$ & $60 \cdot 0 \pm 2 \cdot 0$ \\
\hline Нуро- & $125 \pm 10^{*}$ & $21 \pm 2 *$ & $5 \cdot 9 \pm 0 \cdot 3$ & $0.15 \pm 0.03 *$ & $7 \cdot 0 \pm 0.9^{*}$ \\
\hline Нyро + T3 & $245 \pm 18$ & $44 \pm 3$ & $5 \cdot 6 \pm 0 \cdot 2$ & $1 \cdot 2 \pm 0 \cdot 11^{\dagger}$ & $6 \cdot 0 \pm 0 \cdot 5^{*}$ \\
\hline
\end{tabular}

State 3 and State 4 respiration rates are expressed as $n$ atoms of oxygen consumption $/ \mathrm{min} \times \mathrm{mg}$ of mitochondrial proteins. Respiratory control ratio (RCR), state $3 /$ state 4 respiratory rates. TT3 and TT4 serum levels are expressed as nmol/l. ${ }^{*} P<0 \cdot 05$ vs $\mathrm{N} ;{ }^{+} \mathrm{P}<0 \cdot 05$ vs Hypo.

\section{Effect of T3 on the transcription of liver AQP8}

Semi-quantitative RT-PCR was carried out to evaluate the possible effects of $\mathrm{T} 3$ on the expression of hepatic AQP8 at a transcriptional level. To do that, the mRNA of AQP8 was measured in livers from rats in various thyroid states and compared with the mRNA levels of the housekeeper gene $\beta$-actin that was taken as an internal control.

The livers of hypothyroid rats showed significantly higher levels of AQP8 mRNA $(+82 \%)$ when compared with the levels measured in the livers of the euthyroid counterparts (Fig. 1A and B). T3 administration to hypothyroid rats induced a decrease in the AQP8 transcript of -58 and $-21 \%$ when compared with the hypo- or euthyroid livers respectively (Fig. 1B). These data suggest a negative effect exerted by $\mathrm{T} 3$ on the expression of the $A Q P 8$ gene. Unlike AQP8 in this study and AQP9 in a recent work using porcine liver (Caperna et al. 2007), no significant changes were found at the mRNA level for AQP9, the other major AQP expressed in the rat liver, throughout the various thyroid states (data not shown).

To obtain further insight into the effect of $\mathrm{T} 3$ on $A Q P 8$ gene expression, time-course experiments were also performed. As shown in Fig. 2A and B, AQP8 transcript levels from liver harvested from hypothyroid rats were significantly lower after 6 -h T3 treatment, while they declined by $2 \cdot 5$-fold at 48-h hormonal challenge, reaching values not significantly different from those detected in the livers from hypothyroid rats chronically treated with $\mathrm{T} 3$ (Hypo $+\mathrm{T} 3)$.

\section{Immunoblotting analysis of liver mitochondrial AQP8 in the different thyroid states}

Immunoblotting experiments were performed to look at possible post-transcriptional regulation of AQP8 by T3. Mitochondria were incubated with rabbit polyclonal antibodies directed against an $\mathrm{N}$ terminus peptide of rat AQP8 (Ferri et al. 2003) and the resulting immunoreactivity was analyzed by densitometric analysis.

Consistent with the semi-quantitative RT-PCR studies, the AQP8 immunoreactivity ( $28 \mathrm{kDa}$ band) detected in the mitochondria $(3000 \mathrm{~g}$ fraction) of the hypothyroid livers appeared significantly increased $(+107 \%)$ when compared with the euthyroid counterpart, while $\mathrm{T} 3$ administration to hypothyroid rats strongly decreased protein levels $(-76 \%$; Fig. 3A-C). The nearly appreciable immunoreactivities detected in the plasma membrane $(17000 \boldsymbol{g}$ pellet $)$ and microsomal $(100000 \mathrm{~g}$ pellet) fractions hampered the estimation of the AQP8 expression in such subcellular compartments in the various thyroid states (data not shown).

Immunogold electron microscopy analysis of mitochondrial AQP8 in the various thyroid states

In order to evaluate the submitochondrial distribution as well as the extent of AQP8 expression in the liver of rats in the different thyroid states, an immunogold electron microscopybased morphometric analysis was performed. To do that, we
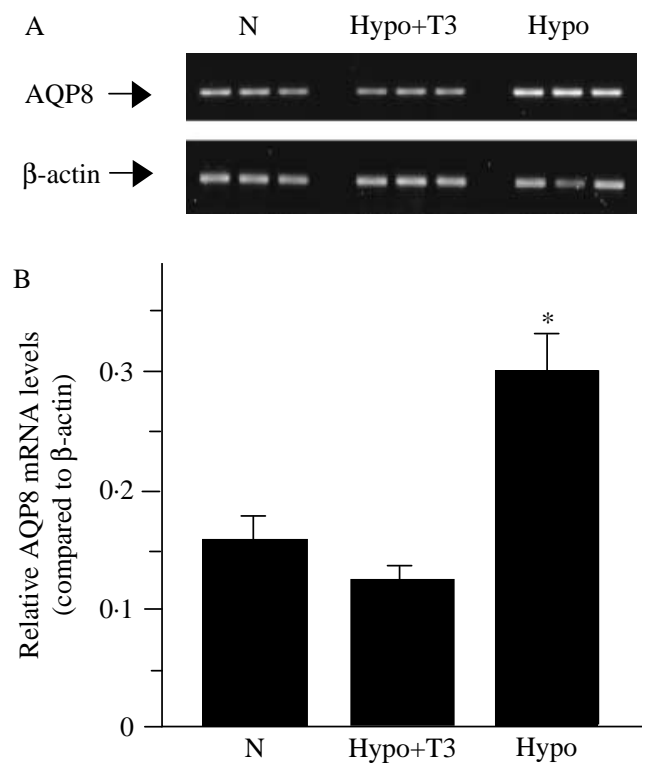

Figure 1 Semi-quantitative RT-PCR analysis of AQP8 mRNA expression in livers of rats in the various thyroid states. (A) Representative experiment showing AQP8 in the livers of the eu-thyroid (N), hyper- (Hypo + T3), and hypothyroid (Hypo) rats. The mRNA of the housekeeping gene $\beta$-actin was taken as internal control. (B) Densitometric analysis of AQP8 mRNA normalized against the $\beta$-actin transcript ( $n=9$ rats). The level of AQP8 mRNA is much higher in the Hypo than in the Hypo $+\mathrm{T} 3$ or $\mathrm{N}$ conditions. ${ }^{*} P<0 \cdot 01$. 
A

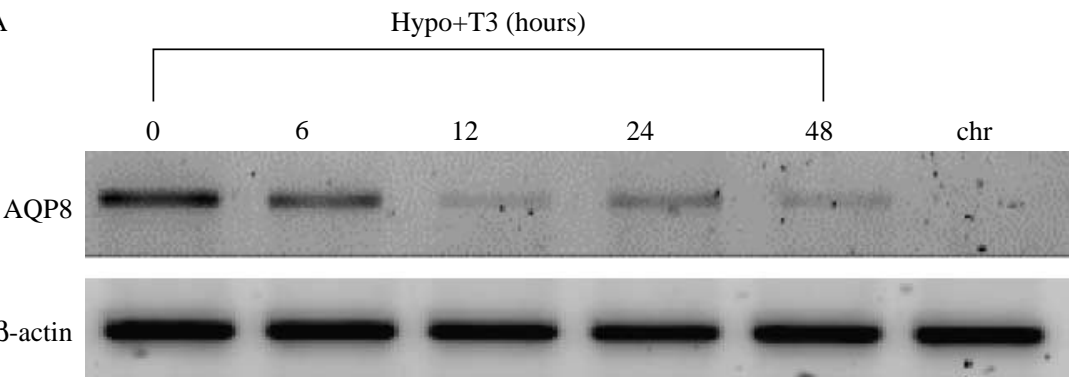

B

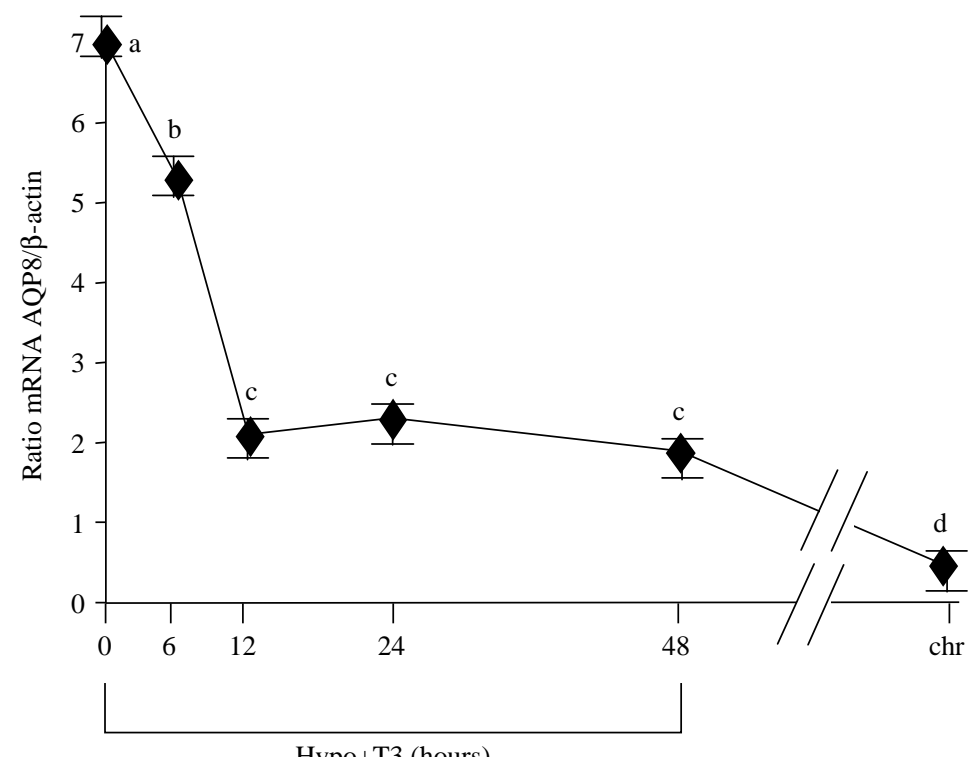

Figure 2 Time-course analysis of hepatic AQP8 mRNA after T3 administration to hypothyroid rats. (A) Each treatment was performed in triplicate, but a representative panel is shown. $\beta$-Actin mRNA levels were measured as the internal standard. (B) Quantification of the data obtained from triplicate RT-PCR measurements. Data are expressed relative to the value obtained for hypo liver, and are presented separately for each time point. Error bars represent s.E. of the mean $(n=3$ rats). chr, chronic (1 week) administration of T3 to hypothryroid rats. Points labeled with dissimilar letters $(\mathrm{a}-\mathrm{d})$ are significantly different $(P<0 \cdot 05)$.

employed samples of the same freshly isolated $3000 \mathrm{~g}$ pellets of mitochondria used for the above immunoblotting experiments.

Confirming our previous immunoelectron microscopy studies (Ferri et al. 2003, Calamita et al. 2005), AQP8 was localized to the inner mitochondrial membrane (Fig. 4A-C). Regardless of the thyroid status, AQP8 reactivity was not homogenously distributed among mitochondria, some of which appeared poorly labeled or even unstained to the AQP8 antibody. However, there were quantitative differences related to thyroid status. Indeed, when AQP8 expression was evaluated morphometrically by counting the number of gold particles over the immunoreactive mitochondria, the levels of mitochondrial AQP8 were higher in the hypothyroid $(+117 \%)$ than in the euthyroid counterpart (Fig. 4E), while T3 administration to hypothyroid rats decreased the levels of AQP8 immunoreactivity by about $-68 \%$. This result was consistent with both the RT-PCR and the immunoblotting studies. No immunogold particles were seen in the control sections where the AQP8 antibodies were omitted (Fig. 4D).

\section{Analysis of the liver IMM water permeability}

Having found regulated expression of AQP8 in the liver mitochondria in the various thyroid states, we then biophysically assessed the water permeability properties of such mitochondria. Using stopped-flow spectrophotometry, we thus directly defined the osmotic water permeability $\left(P_{\mathrm{f}}\right)$ of basically pure and homogeneous IMM vesicles from the $3000 \mathrm{~g}$ mitochondria prepared from the livers in the various thyroid states by measuring the scattered light intensity. IMM vesicles from the intact mitochondria were obtained by sonication from liver mitoplasts and had a mean vesicle diameter of $275 \pm 36 \quad(n=722), 263 \pm 31 \quad(n=639)$, and 


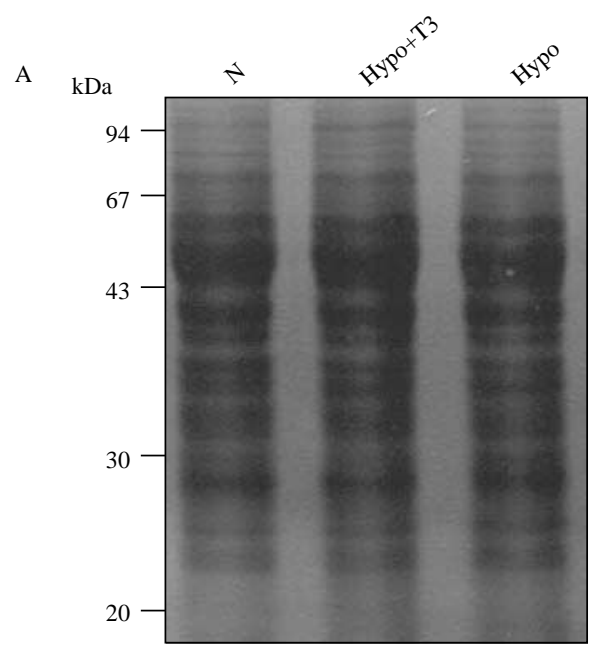

B
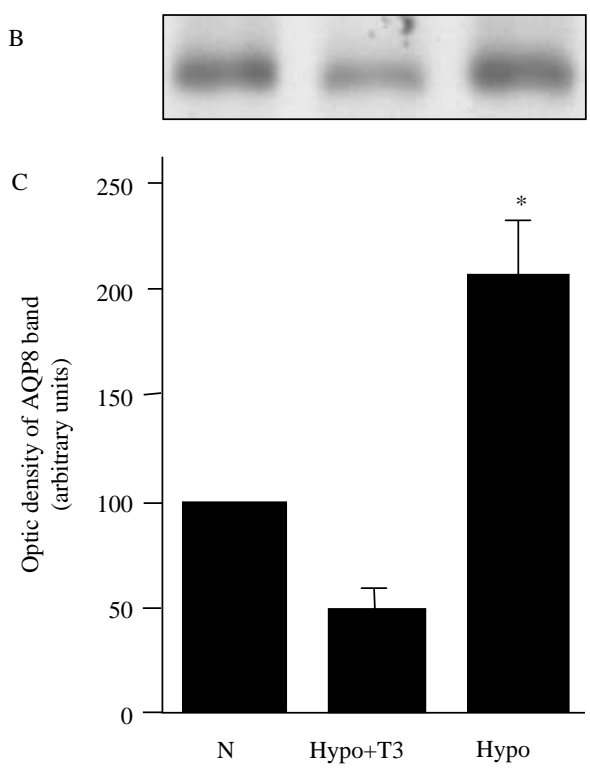

Figure 3 Immunoblotting analysis of AQP8 in the liver in the various thyroid states. (A) Representative blot stained with Coomassie blue used as control for total protein loading. (B) Representative immunoblotting showing the comparative intensity of the $28 \mathrm{kDa}$ AQP8 band revealed in the $3000 \mathrm{~g}$ fraction of liver mitochondria from N, Hypo $+\mathrm{T} 3$, and Hypo rats. (C) Densitometric analysis of the AQP8 immunoreactivity. Data are expressed in arbitrary units as means \pm S.E.M. from eight independent mitochondrial preparations per experimental condition. ${ }^{*} P<0 \cdot 01$.

$282 \pm 34(n=776) \mathrm{nm}$ for the N, Hypo + T3, and Hypo livers respectively. Such diameters were statistically similar in one group vs each of the other two groups. Vesicles were subjected rapidly to a hypertonic osmotic gradient (140 mosM) and the time course of the vesicle shrinkage was followed from the change in scattered light. In line with our previous study (Calamita et al. 2005, 2006), the rate constant $\left(K_{\mathrm{i}}\right)$ of the osmotic equilibration of the IMM vesicles from all three experimental conditions was strikingly high (Fig. 5A).
However, no significant differences were seen among the calculated osmotic permeabilities of the IMM vesicles from the N, Hypo + T3, and Hypo livers (443.1 $\pm 22 \cdot 3,462 \cdot 9 \pm$ $34 \cdot 2$, and $397 \cdot 4 \pm 27 \cdot 6 \mu \mathrm{m} / \mathrm{s}$ respectively; Fig. 5B). No significant differences were also found in the extents of inhibition of IMM osmotic permeability induced by $300 \mu \mathrm{M}$ $\mathrm{HgCl}_{2}$, a compound blocking most AQPs, including AQP8 (Ishibashi et al. 1997, Liu et al. 2006; Fig. 5B). Consistent with the existence of $\mathrm{a}^{2+}{ }^{2+}$-inhibitable aqueous pathway for osmotic water permeability across the IMM, all three inhibitions were reversed after exposure to the reducing agent $\beta$-mercaptoethanol (Fig. 5B). The scattered light did not change in a series of control experiments where vesicles were mixed with isosomotic buffer (data not shown) proving absence of artefacts.

\section{Discussion}

This work reports changes of AQP8 expression in liver mitochondria of rats with different thyroid status. AQP8 mRNA and protein were increased in hypothyroid rats in comparison with control or T3-treated hypothyroid rats. The effect of T3 was relatively fast. Changes in AQP8 expression were also confirmed by direct morphometric analysis of immunogold particles in the electron microscopy. The IMM osmotic permeabilities were found not to be correlated with changes of AQP8 concentration. Data suggest that T3 negatively regulates the $A Q P 8$ gene, probably through a negative thyroid hormone response element, and that control of AQP8 expression may be relevant to the regulation of mitochondrial metabolism by thyroid hormones.

The fact that (1) the levels of both AQP8 transcript and protein were considerably increased in the hypothyroid liver when compared with the euthyroid counterpart and (2) the observation that the $\mathrm{T} 3$ treatment of hypothyroid rats strongly decreased the AQP8 mRNA and protein to levels slightly lower than those measured in euthyroid rats suggest that the downregulation of AQP8 by T3 may well be due to a T3-dependent interaction of thyroid hormone receptor with putative negative thyroid hormone response elements (nTRE; Crone et al. 1990) contained in the AQP8 promoter. This mechanism of regulation is normally used by $\mathrm{T} 3$ when acting on its negatively regulated target genes (Li et al. 2001, Shibusawa et al. 2003). The hypothesis of a T3 regulation mediated by nTREs is consistent with the time-course study showing that the timing of the T3-transcriptional downregulation of AQP8 is short (few hours), as for the other genes negatively modulated by T3 (Yen 2001). The precise mechanism of $\mathrm{T} 3$ regulation of AQP8 will be a matter for future investigation aiming to clone the rat AQP8 promoter and characterize its modulation. Only a minority of the known target genes are negatively regulated by $\mathrm{T} 3$, as they are expressed in the pituitary or hypothalamus (Yen \& Chin 1994, Yen 2001) rather than peripheral tissues. Therefore, together with other 

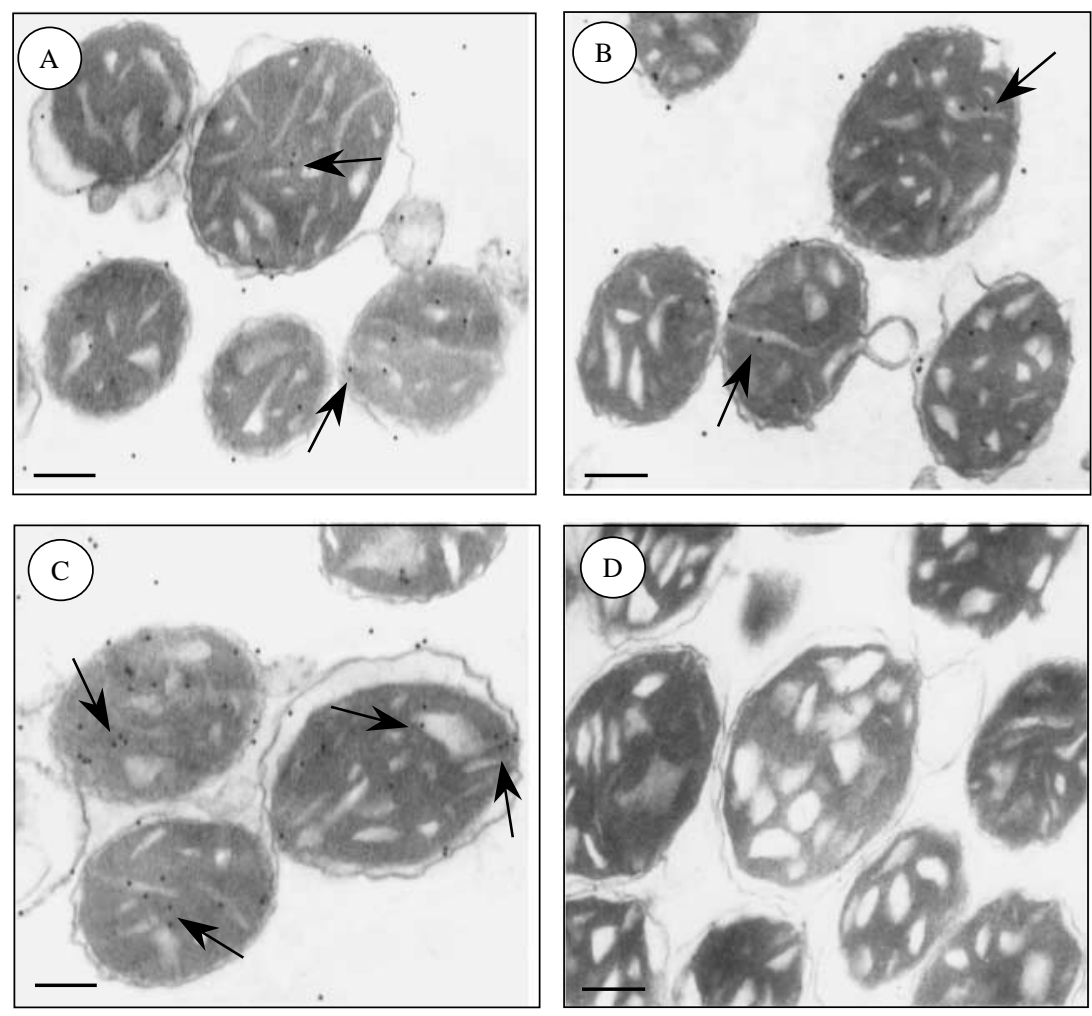

E

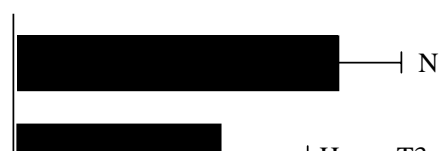

Hypo+T3

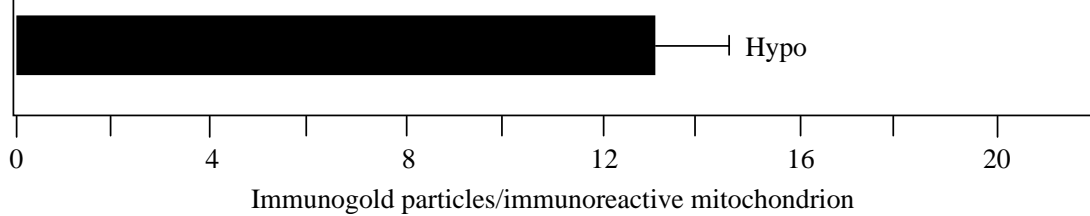

Figure 4 Immunogold electron microscopy of mitochondrial AQP8 expression and distribution in the liver of rats in various thyroid states. Pellets of freshly isolated liver mitochondria (3000 $\mathrm{g}$ fraction) prepared from rat livers at various thyroid states $((\mathrm{A}) \mathrm{N}$; (B) Hypo + T3; (C) Hypo) were analyzed by immunoelectron microscopy as reported in Materials and Methods. Although AQP8 immunogold particles (arrows) are observed in all three experimental conditions, the immunoreactivity observed over the inner mitochondrial membrane is greater in the Hypo mitochondria than in the corresponding $\mathrm{N}$ and Hypo $+\mathrm{T} 3$ samples. (D) Absence of immunoreactivity is seen in the mitochondrial pellets where the primary antibody was omitted (negative control). Bar, $300 \mathrm{~nm}$. (E) Morphometric analysis of the immunogold particle distribution of AQP8 in pellets of freshly isolated rat liver mitochondria in various thyroid states. The AQP8 immunoreactivity corresponds to the number of gold particles over immunoreactive mitochondria. The mean number of AQP8 particles is higher in Hypo than in the $\mathrm{N}$ and Hypo $+\mathrm{T} 3$ counterparts $(P<0 \cdot 01)$.

negatively regulated hepatic genes (Feng et al. 2000), AQP8 may serve as a marker of $\mathrm{T} 3$ action outside the pituitary in assessing the molecular mechanism of negative regulation by nuclear hormone receptors.
The negative modulation exerted by T3 on AQP8 seems not to be a general effect on rat liver AQPs since the hepatic mRNA levels of AQP9, the other major AQP in liver (Huebert et al. 2002), were unchanged in the various thyroid 

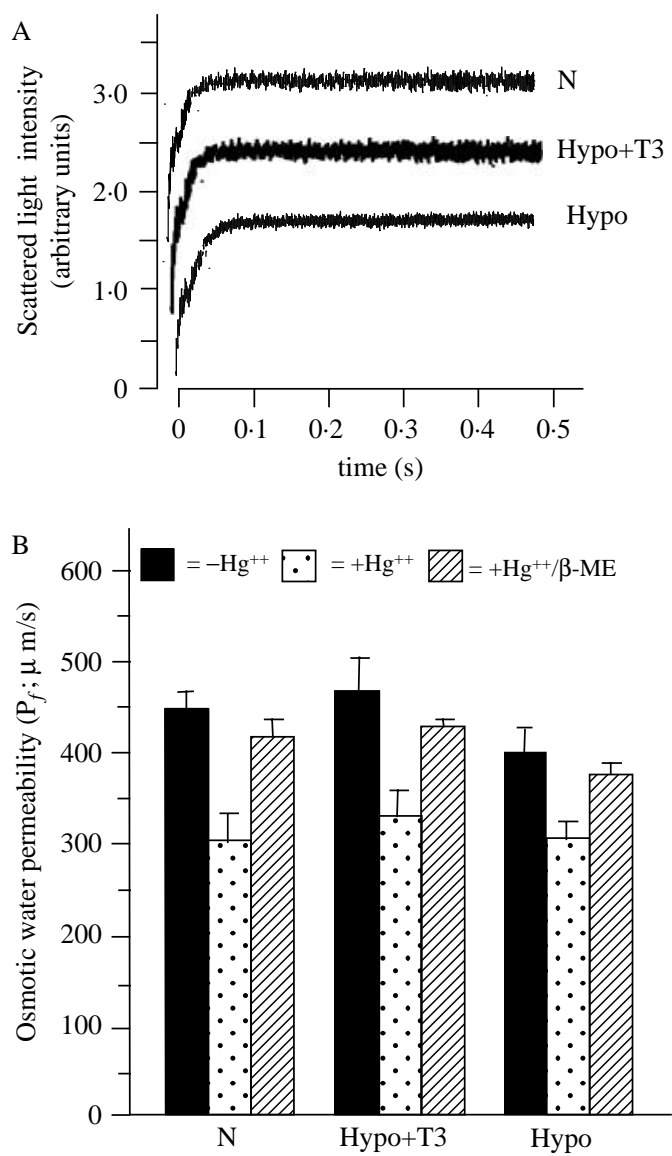

Figure 5 Stopped-flow light scattering analysis of liver IMM osmotic permeability in rats in various thyroid states. (A) Representative experiments of stopped-flow light scattering using IMM vesicles prepared from the $3000 \mathrm{~g}$ subpopulation of liver mitochondria and subject to a hypertonic osmotic gradient of $140 \operatorname{mos} M$ (see Materials and Methods for details). (B) IMM osmotic water permeability and effect of $300 \mu \mathrm{M} \mathrm{HgCl}_{2}$ in rat livers in various thyroid states. The osmotic membrane water permeability $\left(P_{f}\right)$ of the IMM vesicles is extraordinarily high and of comparable extent in the livers under all three experimental conditions (N, Hypo + T3, and Hypo). No significant differences are also observed regarding the inhibitory effect exerted by the $\mathrm{Hg}^{++}$ion $(P>0 \cdot 05)$, a known blocker of the facilitated diffusion of water across membranes, and the related reversal by $10 \mathrm{mM}$ reducing agent $\beta$-mercaptoethanol ( $\beta$-ME). Data are mean values \pm s.E. from three independent vesicle preparations.

states. This idea agrees with the functional and regulatory distinctions known to exist between AQP8 and AQP9 in rat liver (Garcia et al. 2001, Kuriyama et al. 2002). The absence of T3 regulation of AQP9 in rat liver does not confirm the increase in the mRNA levels of AQP9 seen by Caperna et al. (2007) who looked at the expression and distribution of AQP9 in pig liver tissues after stimulation with hormones, such as glucagon, insulin, and T3. Because the T3-induced enhancement of AQP9 transcript seen by Caperna et al. (2007) was not accompanied by a parallel increase in the same $\mathrm{AQP}$ at a protein level, it is conceivable to speculate that the
T3 modulation of AQP9 in porcine liver is not of major physiological relevance. As also concluded by the same authors who found that glucagon enhanced the absolute levels of AQP9 protein (in addition to the mRNA ones), in pig liver AQP9 may be primarily responsive to glucagon induction.

Another important question arising from the present work regards the functional meaning of the $\mathrm{T} 3$ modulation of AQP8 in liver mitochondria. Because of its high water conductivity (Ishibashi et al. 1997, Liu et al. 2006) and localization in the inner mitochondrial membrane (Ferri et al. 2003, Calamita et al. 2005), it is tempting to speculate that the effect of T3 on AQP8 expression may be a way by which thyroid hormone participates in the morphological modifications observed in mitochondria of tissues from animals in different thyroid states. Changes in mitochondrial volume may also have consequences on mitochondrial metabolic activities/efficiency (Garlid 1988, Kaasik et al. 2006) and may participate in mechanical-signaling pathways (Safiulina et al. 2006). Indeed, inhibitors of the electron transport chain lead to shrinkage of the mitochondrial matrix (Mustafa et al. 1966, Hackenbrock 1968). However, although the idea of T3 modulation of mitochondrial shape involving the AQP8 water channel is consistent with the observed increase in mitochondrial volume occurring in hypothyroid conditions (Goglia et al. 1989), it contrasts with the fact that the overall liver IMM osmotic water permeability and the related extent of $\mathrm{Hg}^{++}$-inhibition are unchanged in the various thyroid states. The inconsistency may be only apparent, due to the fact that, as indicated by our recent biophysical study (Calamita et al. 2006), pathways other than AQP8 may be important for mitochondrial water permeability, such as the mitochondrial transition pore (MTP). A narrow interplay between mitochondrial volume and MTP is also indicated by the fact that decreasing mitochondrial volume alters the activity of several MTP regulators (Nogueira et al. 2005). As hypothyroidism is known to render liver mitochondria resistant to the opening of MTP (Chavez et al. 1998), while in vivo thyroid hormone treatment has been reported to induce the opening of MTP (Kalderon et al. 1995), a compensatory mechanism through AQP8 may exist in hypothyroid mitochondria to allow mitochondrial volume homeostasis. This could explain why no differences are observed between the overall osmotic water permeabilities of the liver IMMs of Hypo and Hypo + T3 rats. Alternative meanings for the T3 regulation of AQP8 in rat liver, such as being one of the mechanisms by which $\mathrm{T} 3$ modulates the amino acid metabolism, cannot be ruled out since AQP8 has been reported to be permeable to the $\mathrm{NH}_{4}^{+}$ion in addition to water (Jahn et al. 2004, Holm et al. 2005, Liu et al. 2006, Yang et al. 2006b). This possibility is in line with the fact that (1) hypothyroidism is associated with increased hepatic capacity to synthesize urea in rats, and thus increased ammonia production (Marti et al. 1988, Hayase et al. 1991) and (2) our recent work of proteomics on rat liver showing that hyperthyroidism leads to downregulation of ornithine carbamoyltransferase and arginase-1 (Silvestri et al. 2006), 
two enzymes underlying the urea cycle and the arginine degradation pathway respectively.

In summary, the present work shows that triiodothyronine modulates the expression of AQP8 in rat liver mitochondria. The T3 action on AQP8 is exerted at a transcriptional level and already occurs in vivo a few hours after T3 injection. Future studies will be addressed to evaluate the functional meaning of the thyroid regulation of AQP8, such as influencing the mitochondrial shape and/or controlling the amino acid metabolism by acting on the urea cycle.

\section{Acknowledgements}

The skilful and valuable contribution from Prof Giuseppa E Liquori and Dr Maria Mastrodonato to the electron microscopy studies is gratefully acknowledged.

\section{Funding}

This work was supported by the Italian Programmi di Ricerca Scientifica di Rilevante Interesse Nazionale (PRIN; grant no. 2004053441) to G C and F G and Centro di Eccellenza di Genomica in Campo Biomedico ed Agrario (CEGBA) to $\mathrm{GC}$ and M S. This work does not have any conflict of interest that would prejudice its impartiality.

\section{References}

Beavis AD, Brannan RD \& Garlid KD 1985 Swelling and contraction of the mitochondrial matrix. I. A structural interpretation of the relationship between light scattering and matrix volume. Journal of Biological Chemistry 260 13424-13433.

Calamita G, Ferri D, Gena P, Liquori GE, Cavalier A, Thomas D \& Svelto M 2005 The inner mitochondrial membrane has aquaporin- 8 water channels and is highly permeable to water. Journal of Biological Chemistry $\mathbf{2 8 0}$ 17149-17153.

Calamita G, Gena P, Meleleo D, Ferri D \& Svelto M 2006 Water permeability of rat liver mitochondria: a biophysical study. Biochimica et Biophysica Acta 1758 1018-1024.

Caperna TJ, Shannon AE, Richards MP, Garrett WM \& Talbot NC 2007 Identification and characterization of aquaporin-9 (AQP9) in porcine hepatic tissue and hepatocytes in monolayer culture. Domestic Animal Endocrinology.

Chavez E, Franco M, Reyes-Vivas H, Zazueta C, Ramirez J \& Carrillo R 1998 Hypothyroidism renders liver mitochondria resistant to the opening of membrane permeability transition pore. Biochimica et Biophysica Acta 1407 243-248.

Crone DE, Kim HS \& Spindler SR 1990 Alpha and beta thyroid hormone receptors bind immediately adjacent to the rat growth hormone gene TATA box in a negatively hormone-responsive promoter region. Journal of Biological Chemistry 265 10851-10856.

Estabrook R 1967 Mitochondrial respiratory control and the polarographic measurement of ADP/O ratios. Methods in Enzymology 10 41-47.

Feng X, Jiang Y, Meltzer P \& Yen PM 2000 Thyroid hormone regulation of hepatic genes in vivo detected by complementary DNA microarray. Molecular Endocrinology 14 947-955.

Ferri D, Mazzone A, Liquori GE, Cassano G, Svelto M \& Calamita G 2003 Ontogeny, distribution, and possible functional implications of an unusual aquaporin, AQP8, in mouse liver. Hepatology 38 947-957.
Freake HC, Schwartz HL \& Oppenheimer JH 1989 The regulation of lipogenesis by thyroid hormone and its contribution to thermogenesis. Endocrinology 125 2868-2874.

Garcia F, Kierbel A, Larocca MC, Gradilone SA, Splinter P, LaRusso NF \& Marinelli RA 2001 The water channel aquaporin-8 is mainly intracellular in rat hepatocytes, and its plasma membrane insertion is stimulated by cyclic AMP. Journal of Biological Chemistry 276 12147-12152.

Garlid KD 1988 Mitochondrial volume control. In: Integration of mitochondrial function. Eds CR Hackenbrock, JJ Lemasters, RG Thurman \& HVV Westerhoff pp 257-276 New York: Plenum Press.

Goglia F, Liverini G, Lanni A \& Barletta A 1988 Mitochondrial DNA, RNA and protein synthesis in normal, hypothyroid and mildly hyperthyroid rat liver during cold exposure. Molecular and Cellular Endocrinology 55 141-147.

Goglia F, Liverini G, Lanni A, Iossa S \& Barletta A 1989 The effect of thyroid state on respiratory activities of three rat liver mitochondrial fractions. Molecular and Cellular Endocrinology 62 41-46.

Goglia F, Moreno M \& Lanni A 1999 Action of thyroid hormones at the cellular level: the mitochondrial target. FEBS Letters 452 115-120.

Gross NJ 1971 Control of mitochondrial turnover under the influence of thyroid hormone. Journal of Cell Biology 48 29-40.

Hackenbrock CR 1968 Ultrastructural bases for metabolically linked mechanical activity in mitochondria. II. Electron transport-linked ultrastructural transformations in mitochondria. Journal of Cell Biology 37 345-369.

Hayase K, Yonekawa G, Yokogoshi H \& Yoshida A 1991 Triiodothyronine administration affects urea synthesis in rats. Journal of Nutrition $121970-978$.

van Heeswijk MP \& van Os CH 1986 Osmotic water permeabilities of brush border and basolateral membrane vesicles from rat renal cortex and small intestine. Journal of Membrane Biology 92 183-193.

Holm LM, Jahn TP, Moller AL, Schjoerring JK, Ferri D, Klaerke DA \& Zeuthen $\mathrm{T} 2005 \mathrm{NH}_{3}$ and $\mathrm{NH}_{4}^{+}$permeability in aquaporin-expressing Xenopus oocytes. Pflügers Archiv 450 415-428.

Huebert RC, Splinter PL, Garcia F, Marinelli RA \& LaRusso NF 2002 Expression and localization of aquaporin water channels in rat hepatocytes. Evidence for a role in canalicular bile secretion. Journal of Biological Chemistry 277 22710-22717.

Ishibashi K, Kuwahara M, Kageyama Y, Tohsaka A, Marumo F \& Sasaki S 1997 Cloning and functional expression of a second new aquaporin abundantly expressed in testis. Biochemical and Biophysical Research Communications 237 714-718.

Jahn TP, Moller AL, Zeuthen T, Holm LM, Klaerke DA, Mohsin B, Kuhlbrandt W \& Schjoerring JK 2004 Aquaporin homologues in plants and mammals transport ammonia. FEBS Letters 574 31-36.

Jakovcic S, Swift HH, Gross NJ \& Rabinowitz M 1978 Biochemical and stereological analysis of rat liver mitochondria in different thyroid states. Journal of Cell Biology 77 887-901.

Kaasik A, Safiulina D, Zharkovsky A \& Veksler V 2006 Regulation of mitochondrial matrix volume. American Journal of Physiology. In Press.

Kalderon B, Hermesh O \& Bar-Tana J 1995 Mitochondrial permeability transition is induced by in vivo thyroid hormone treatment. Endocrinology 136 3552-3556.

Kuriyama H, Shimomura I, Kishida K, Kondo H, Furuyama N, Nishizawa H, Maeda N, Matsuda M, Nagaretani H, Kihara S et al. 2002 Coordinated regulation of fat-specific and liver-specific glycerol channels, aquaporin adipose and aquaporin 9. Diabetes 51 2915-2921.

Lanni A, Moreno M, Lombardi A \& Goglia F 1996 Calorigenic effect of diiodothyronines in the rat. Journal of Physiology 494 831-837.

Lee WK, Bork U, Gholamrezaei F \& Thevenod F 2005 Cd(2+)-induced cytochrome $\mathrm{c}$ release in apoptotic proximal tubule cells: role of mitochondrial permeability transition pore and $\mathrm{Ca}(2+)$ uniporter. American Journal of Physiology. Renal Physiology 288 F27-F39.

Li QL, Jansen E, Brent GA \& Friedman TC 2001 Regulation of prohormone convertase 1 (PC1) by thyroid hormone. American Journal of Physiology. Endocrinology and Metabolism 280 E160-E170.

Liu K, Nagase H, Huang CG, Calamita G \& Agre P 2006 Purification and functional characterization of aquaporin-8. Biology of the Cell 98 153-161. 
Marti J, Portoles M, Jimenez-Nacher I, Cabo J \& Jorda A 1988 Effect of thyroid hormones on urea biosynthesis and related processes in rat liver. Endocrinology 123 2167-2174.

Moreno M, Lanni A, Lombardi A \& Goglia F 1997 How the thyroid controls metabolism in the rat: different roles for triiodothyronine and diiodothyronines. Journal of Physiology 505 529-538.

Moreno M, Lombardi A, De Lange P, Silvestri E, Ragni M, Lanni A \& Goglia F 2003 Fasting, lipid metabolism, and triiodothyronine in rat gastrocnemius muscle: interrelated roles of uncoupling protein 3 , mitochondrial thioesterase, and coenzyme Q. FASEB Journal 17 1112-1114.

Mulder J, Haddad MN, Vernon K, Baum M \& Quigley R 2003 Hypothyroidism increases osmotic water permeability $(\mathrm{Pf})$ in the developing renal brush border membrane. Pediatric Research 53 1001-1007.

Mustafa MG, Utsumi K \& Packer L 1966 Damped oscillatory control of mitochondrial respiration and volume. Biochemical and Biophysical Research Communications 24 381-385.

Nogueira V, Devin A, Walter L, Rigoulet M, Leverve X \& Fontaine E 2005 Effects of decreasing mitochondrial volume on the regulation of the permeability transition pore. Journal of Bioenergetics and Biomembranes 37 25-33.

Paradies G, Ruggiero FM, Petrosillo G \& Quagliariello E 1994 Enhanced cytochrome oxidase activity and modification of lipids in heart mitochondria from hyperthyroid rats. Biochimica et Biophysica Acta 1225 165-170.

Roodyn DB 1965 Further study of factors affecting amino acid incorporation into protein by isolated mitochondria. Biochemical Journal 97 782-793.

Safiulina D, Veksler V, Zharkovsky A \& Kaasik A 2006 Loss of mitochondrial membrane potential is associated with increase in mitochondrial volume: physiological role in neurones. Journal of Cellular Physiology 206 347-353.

Schonfeld P, Wieckowski MR \& Wojtczak L 1997 Thyroid hormoneinduced expression of the ADP/ATP carrier and its effect on fatty acidinduced uncoupling of oxidative phosphorylation. FEBS Letters 416 19-22.
Shibusawa N, Hollenberg AN \& Wondisford FE 2003 Thyroid hormone receptor DNA binding is required for both positive and negative gene regulation. Journal of Biological Chemistry 278 732-738.

Silvestri E, Moreno M, Schiavo L, de Lange P, Lombardi A, Chambery A, Parente A, Lanni A \& Goglia F 2006 A proteomics approach to identify protein expression changes in rat liver following administration of $3,5,3^{\prime}$ triiodo-L-thyronine. Journal of Proteome Research 5 2317-2327.

Soboll S 1993 Thyroid hormone action on mitochondrial energy transfer. Biochimica et Biophysica Acta 1144 1-16.

Soboll S, Conrad A \& Hebisch S 1994 Influence of mitochondrial creatine kinase on the mitochondrial/extramitochondrial distribution of high energy phosphates in muscle tissue: evidence for a leak in the creatine shuttle. Molecular and Cellular Biochemistry 133-134 105-113.

Yang B, Zhao D \& Verkman AS 2006a Evidence against functionally significant aquaporin expression in mitochondria. Journal of Biological Chemistry 281 16202-16206.

Yang B, Zhao D, Solenov E \& Verkman AS $2006 b$ Evidence from knockout mice against physiologically significant aquaporin 8-facilitated ammonia transport. American Journal of Physiology. Cell Physiology 291 C417-C423.

Yen PM 2001 Physiological and molecular basis of thyroid hormone action. Physiological Reviews 81 1097-1142.

Yen PM \& Chin WW 1994 Molecular mechanisms of dominant negative activity by nuclear hormone receptors. Molecular Endocrinology 8 1450-1454.

Received in final form 15 October 2006

Accepted 17 October 2006

Made available online as an Accepted Preprint 30 October 2006 